\title{
Surrogate models of Pareto-optimal planar inductors
}

\author{
Authors Name/s per 1st Affiliation (Author) \\ line 1 (of Affiliation): dept. name of organization \\ line 2: name of organization, acronyms acceptable \\ line 3: City, Country \\ line 4: e-mail address if desired
}

\author{
Authors Name/s per 2nd Affiliation (Author) \\ line 1 (of Affiliation): dept. name of organization \\ line 2: name of organization, acronyms acceptable \\ line 3: City, Country \\ line 4: e-mail address if desired
}

\begin{abstract}
Systematic design methodologies for wireless transceivers require an efficient design of integrated inductors. Early availability of feasible trade-offs between inductance, quality factor and area, is a key enabler towards the improvement of such design methodologies. This paper introduces such an approach in two steps. First, a Pareto-optimal performance front of integrated inductors is obtained by embedding an electromagnetic simulator into a multi-objective optimization tool. Then, starting from the obtained optimal samples, a surrogate model of the performance front is obtained. Experimental results in a $0.35 \mu \mathrm{m}$ CMOS technology are provided.
\end{abstract}

Keywords-RF circuit design; integrated planar inductors; performance modeling; multi-objective optimization; surrogate modeling.

\section{INTRODUCTION}

Increasing integration of wireless transceivers has motivated a growing need for accurate modeling and efficient design of integrated inductors in radiofrequency (RF) circuits. Inductor performances are limited by numerous parasitic effects and their accurate evaluation is a difficult and computationally expensive process. Therefore, designers often resort to the libraries of inductors provided by silicon foundries. However, such inductor libraries have usually a very limited offer and the design of RF blocks is conditioned by the available inductors.

High-performance RF circuit design benefits from inductors specifically designed for the application at hand. When addressing the design of RF circuits including inductors, the designer is faced with questions like: Is this inductance realizable with this technology and at this frequency of operation? Which is the quality factor that can be obtained with this topology? Which is the required inductor area to achieve a certain inductance and quality factor?

A first common approach is to use analytical equations or models relating design parameters with inductor performances [1],[2]. Due to the large inaccuracies of these models, high performance design requires lengthy iterations between layout implementation and detailed electromagnetic simulation. Even if the approach is successful, just a single, suboptimal design is obtained as the design space exploration implied by the questions posed above can hardly be accomplished.
To address this objective, this paper proposes a methodology in two steps. In the first step, a Pareto-optimal front of inductors exhibiting optimal performance trade-offs between the desired design objectives is obtained. In the second step, a surrogate model relating the performance objectives along the Pareto-optimal front is obtained.

The paper is organized as follows. Section II introduces two basic mathematical tools used to implement our approach, which is described in Section III. Experimental results are shown in Section IV.

\section{MATHEMATICAL INSTRUMENTS}

\section{A. Multi-objective optimization}

Multi-objective optimization is a useful tool to explore trade-offs in situations in which two or more mutually conflicting objectives have to be minimized or maximized.

A multi-objective optimization problem can be mathematically formulated as:

$$
\begin{array}{|cl}
\text { Minimize } \boldsymbol{f}(\boldsymbol{x}) ; & \boldsymbol{f}(\boldsymbol{x}) \in R^{k} \\
\text { such that: } & \boldsymbol{g}(\boldsymbol{x}) \leq 0 ; \quad \boldsymbol{g}(\boldsymbol{x}) \in R^{m} \\
\text { where } x_{L i} \leq x_{i} \leq x_{U i}, & i \in[1, p]
\end{array}
$$

Given the minimization problem in (1), a solution, $\boldsymbol{x}_{A}$, is said to dominate another solution, $\boldsymbol{x}_{B}$, if $\boldsymbol{f}\left(\boldsymbol{x}_{A}\right) \leq \boldsymbol{f}\left(\boldsymbol{x}_{B}\right)$ and the " $<$ " relation verifies for at least one function $i$. Solution $\boldsymbol{x}_{A}$ is said to be non-dominated if no other solution dominates it. The non-dominated set of points of the feasible objective space is usually called the Pareto front and its image in the search space is the Pareto set.

Multi-objective optimization algorithms to solve this problem continue to be a topic of intense research and numerous approaches have been reported: NSGA-II [3], MOEA/D [4], etc.

\section{B. Surrogate modeling}

Surrogate models refer to replacing complex systems whose performance evaluation is computationally expensive by a simpler, although less accurate, model that can be much more efficiently evaluated. 
The problem of surrogate model generation usually involves two aspects. The first one is the selection of sample vectors to build the model. A wide variety of techniques are available, ranging from classical Monte Carlo (MC) sampling, to Latin Hypercube Sampling (LHS) and Quasi Monte-Carlo (QMC) techniques. The second aspect concerns the core functions used to build the model. Literature survey show approaches based on neural networks, support vector machines, Kriging basis functions, etc. As can be easily understood, the accuracy of the model is essential. The error between the outcome of the surrogate model at a new input vector and the accurate evaluation of the complex system is usually measured by the root mean square error. Some techniques, like Kriging models, enable not only obtaining a predicted value for new input vectors but also an estimation of the error. Tools to build Kriging and other surrogate models are available in Matlab toolboxes like SUMO [5] and DACE [6].

\section{PROPOSED APPROACH}

The proposed approach consists of two basic steps and is illustrated in Fig. 1. In the first one, a Pareto optimal front of the performance objectives is generated. In the second one, a surrogate model of the Pareto-optimal front is generated.

The Pareto front generation is performed by using an iterative optimization procedure. The optimization algorithm used in our implementation is the population-based multiobjective optimization algorithm NSGA-II [3]. Inputs to the algorithm include definition of search space, objectives and constraints. At each generation of the optimization algorithm, mutation, selection and crossover operators provide new sets of inductor design parameters: number of turns, turn widths, turns spacing and inner diameter. The layout of each trial inductor is generated by instancing a parameterized layout cell. The layout of different inductor topologies, shown in Fig. 2, has been coded and implemented in the Cadence design environment using SKILL language. The instanced layouts are exported in GDSII format and transformed into a layout format compatible with the Agilent ADS design environment. Such instances are then simulated with the electromagnetic simulator ADS Momentum. The result of one of these simulations is illustrated in Fig. 3. Based on these simulation results, objectives and constraints are evaluated, and used in the ranking procedure of the optimization algorithm. The optimization loop is iteratively applied until a certain number of iterations are executed or a

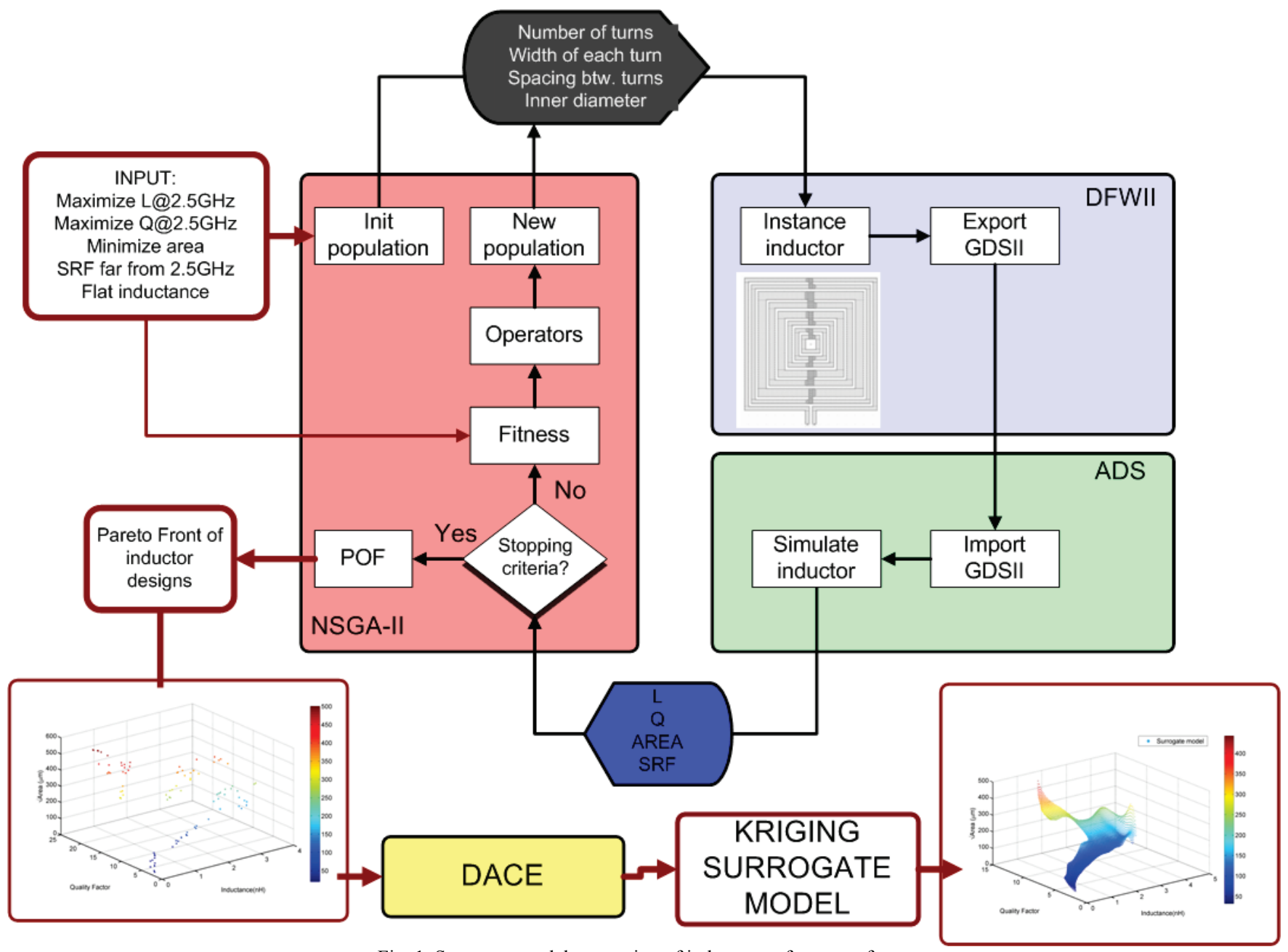

Fig. 1. Surrogate model generation of inductor performance front. 


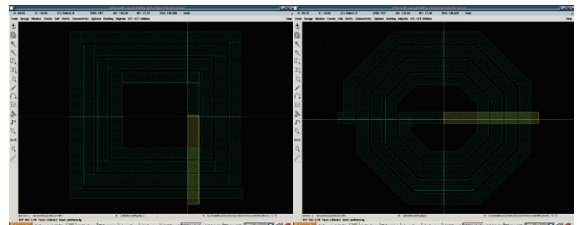

(a)

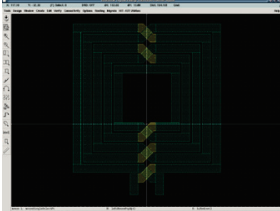

(c)

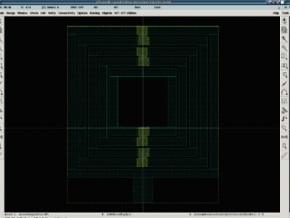

(d)

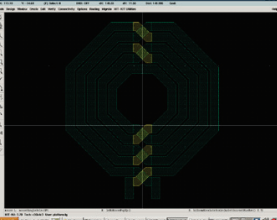

(e)

Fig. 2. Library of parameterized inductor layout cells.

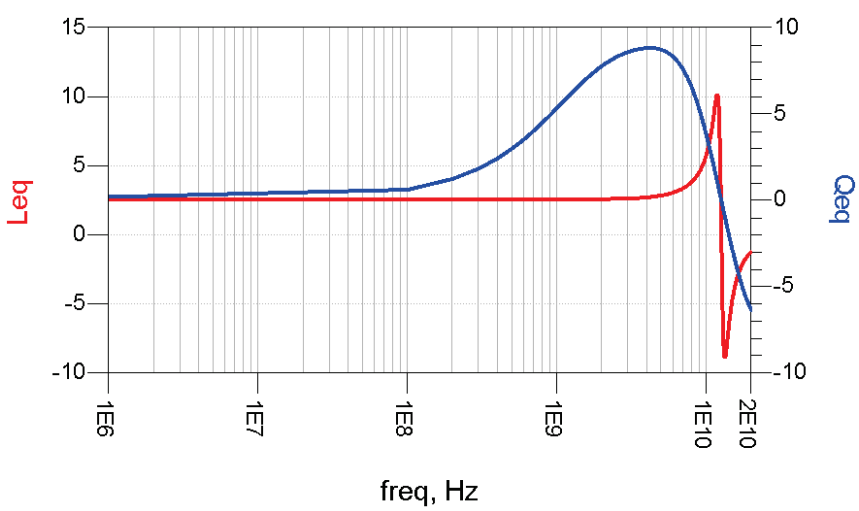

Fig. 3. Equivalent inductance and quality factor.

sufficient convergence to the Pareto front is achieved.

The output of the first step is a set of points of the Pareto front and its corresponding Pareto set, as shown in Fig. 1. This information is already very useful and can be used for automated design purposes, like the design of low noise amplifiers. But it is not able to answer questions like those posed in the introduction of this paper. To address this issue, the second step uses the points of the Pareto front (defined in the objective space) to generate a surrogate model mapping some objectives to some other ones. Kriging surrogate models have been used for this generation.

\section{EXPERIMENTAL RESULTS}

The proposed approach will be used to explore the optimal trade-offs of symmetrical square inductors in a $0.35 \mu \mathrm{m}$ CMOS technology. The number of turns is allowed to vary between 1 and 10 , the turn width between $5 \mu \mathrm{m}$ (the minimum value allowed by the technology) and $100 \mu \mathrm{m}$ and the inner diameter between $10 \mu \mathrm{m}$ and $400 \mu \mathrm{m}$. The turn spacing is fixed at the minimum value: $2.5 \mu \mathrm{m}$, as no performance improvement is theoretically expected from a larger spacing. There are three objectives: area minimization and maximization of quality factor and inductance at $2.5 \mathrm{GHz}$. Constraints are imposed to guarantee that the self-resonant frequency is sufficiently above $2.5 \mathrm{GHz}$ and the inductance is sufficiently flat from dc to slightly above the operating frequency.

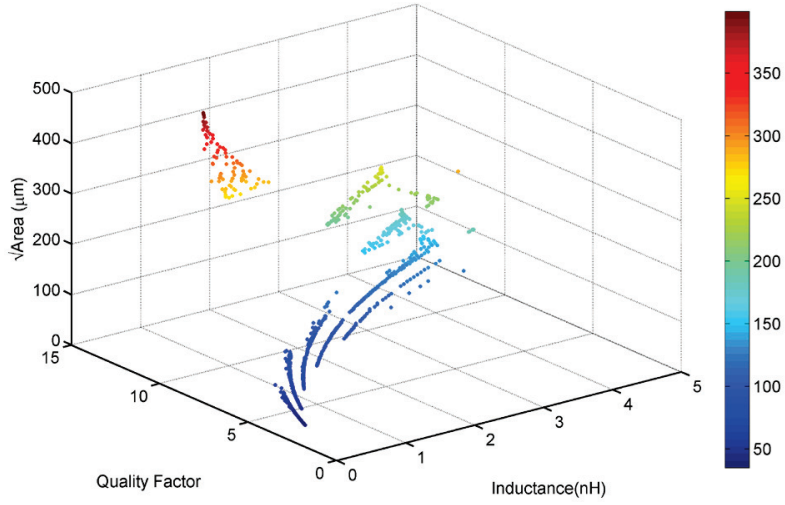

Fig. 4. Inductance vs. quality factor vs. area for symmetrical square inductor.

The application of the first step of the methodology in Fig. 1 provides the Pareto-optimal front of inductance, quality factor and area [7]. As can be seen in Fig. 4, the samples of the Pareto-optimal performance front already give useful information about the trade-offs between inductance, quality factor and area, and have been used in bottom-up synthesis methodologies [8]. However, exploration of trade-offs is necessarily limited by the number of points of the front. Obviously, generation time increases with the number of points.

Detailed trade-off information can be obtained if a surrogate model is obtained. No technique for sample vector selection is applied in this case as the input samples to generate the surrogate model are the samples of the Pareto front obtained in the previous step. The DACE toolbox [6] was used to generate the Kriging surrogate model of the area as a function of inductance and quality factor. The surrogate model so generated can be used for instance to graphically represent the trade-offs between inductance, quality factor and area. A graphical representation requires sweeping a range of values of inductance and quality factor to obtain the corresponding area. But as can be easily checked in Fig. 4, not all pairs of inductance - quality factor values are feasible. This is reflected in a large mean square error of the value estimated by the surrogate model. Fig. 5 shows the estimated error when the

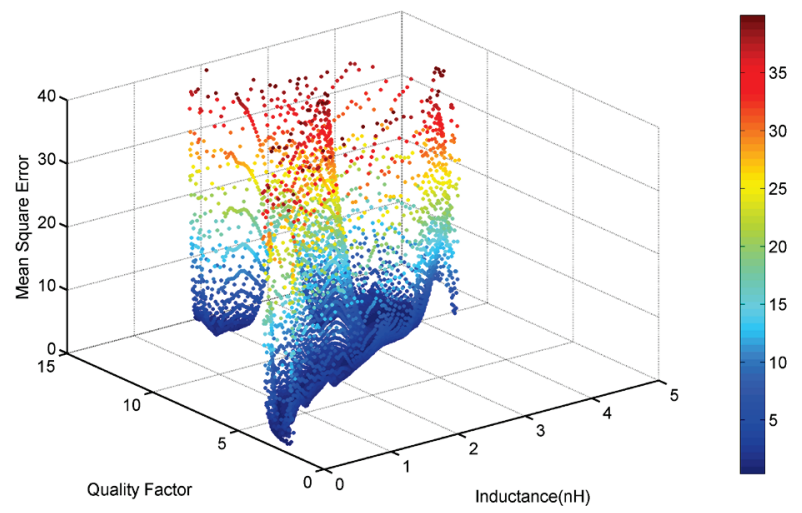

Fig. 5. Error estimation 


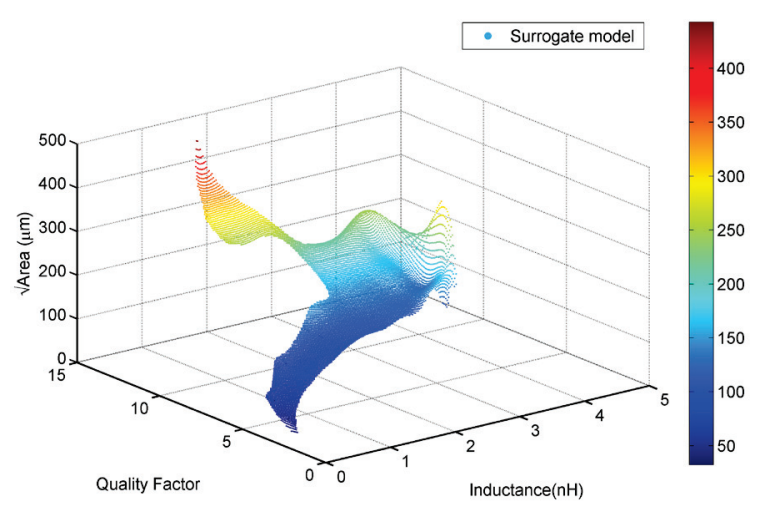

Fig. 6. Graphical representation of surrogate model

inductance and quality factor are swept in the same ranges than in Fig. 4.

The values estimated by the surrogate model can be meaningfully plotted when the estimated error is below a given threshold, as shown in Fig. 6.

The surrogate model can also be evaluated to answer questions like those in the introduction. For instance, the surrogate model can be evaluated for a given inductance value to obtain the trade-off of quality factor and area. For illustration's sake, the area vs. quality factor trade-off is plotted in Fig. 7 for an inductance of $2 \mathrm{nH}$ and in Fig. 8 for $3 \mathrm{nH}$. The same figures also plot the estimation error, showing that such error is small where inductance and quality factor values are
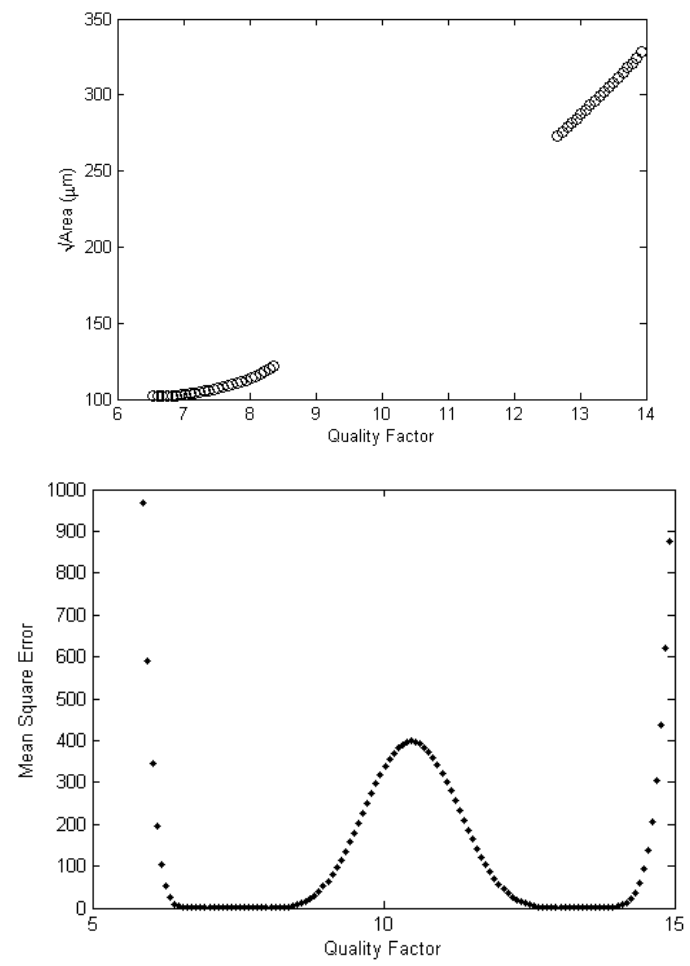

Fig. 7. Graphical representation of area vs. quality factor of surrogate model and estimated error for $\mathrm{L}=2 \mathrm{nH}$. feasible.

\section{REFERENCES}

[1] J. R. Long, and M. A. Copeland, "The modeling, characterization, and design of monolithic inductors for silicon RF IC's", IEEE Journal of Solid_State Circuits, Vol. 32, No. 3, pp. 357-369, March 1997

[2] J. Sieiro, J.M. Lopez-Villegas, J. Cabanillas, J.A. Osorio and J. Samitier, "A physical frequency-dependent compact model for RF integrated inductors", IEEE Trans. on Microwave Theory and Techniques, Vol. 50, No. 1, pp. 384-392, Jan. 2002

[3] K. Deb, A. Pratap, S. Agarwal and T. Meyarivan, "A fast and elitist multiobjective genetic algorithm: NSGA-II," IEEE Trans. on Evolutionary Computation, Vol. 6, No. 2, pp. 182-197, April 2002.

[4] Q. Zhang and H. Li, "MOEA/D: A multiobjective evolutionary algorihtm based on decomposition," IEEE Trans. on Evolutionary Computation, Vol. 11, No. 6, pp. 712-731, Dec. 2007.

[5] Surrogate Modeling (SUMO) Toolbox [online]. http://www.sumo.intec.ugent.be/?q=sumo_toolbox

[6] DACE: A Matlab Kriging toolbox [online]. http://www2.imm.dtu.dk/ hbn/dace/.

[7] J. Esteban-Muller, R. González-Echevarría, C. Sánchez-López, R Castro-López, E. Roca, F.V. Fernández, , J.M. López-Villegas, J. Sieiro and N. Vidal, "Multi-objective optimization of planar inductors," Proc. Int. Workshop on Symbolic and Numerical Methods, Modeling and Applications to Circuit Design ( $\left.\mathrm{SM}^{2} \mathrm{ACD}\right)$, Gammarth (Tunisia), 2010.

[8] C. Sánchez-López, R. Castro-López, E. Roca, F.V. Fernández, R Gonzalez-Echevarría, J. Esteban-Muller, J.M. López-Villegas, J. Sieiro and N. Vidal, "A bottom-up approach to the systematic design of LNAs using evolutionary computation," Proc. Int. Workshop on Symbolic and Numerical Methods, Modeling and Applications to Circuit Design (SM $\left.{ }^{2} \mathrm{ACD}\right)$, Gammarth (Tunisia), 2010.
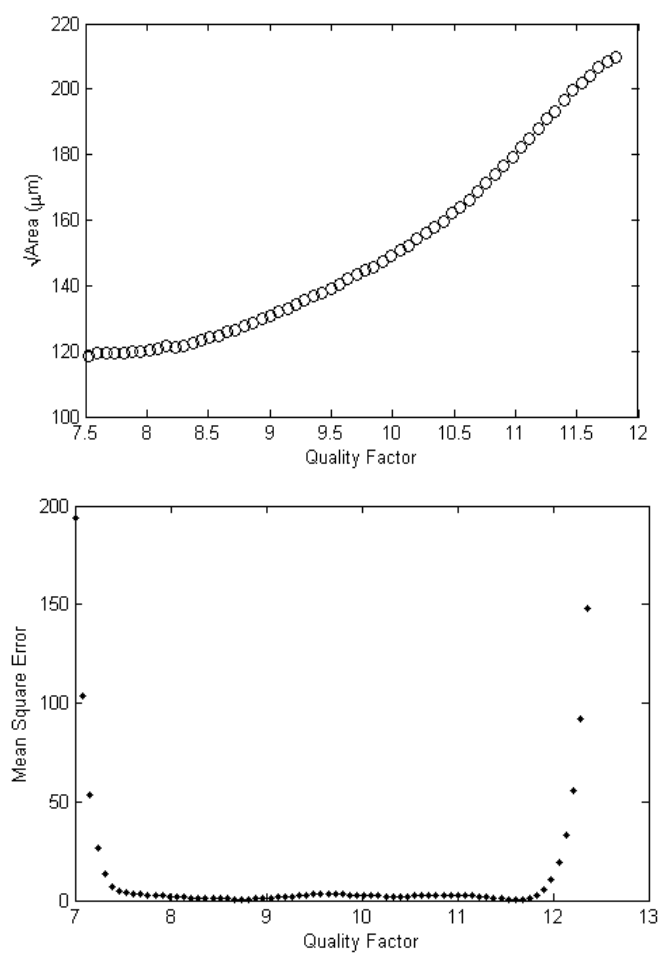

Fig. 8. Graphical representation of area vs. quality factor of surrogate model and estimated error for $\mathrm{L}=3 \mathrm{nH}$. 